Julio- Diciembre 2017

Revista de Investigación

Año 10.

Académica sin Frontera

Núm. 26

ISSN: 2007-8870

http://revistainvestigacionacademicasinfrontera.com

Recibido el 25 de abril de 2017. Dictaminado favorablemente mediante arbitraje el 19 de septiembre de 2017.

\title{
Factores que influyen en la elección del servicio en una estética en los Navojoenses
}

\author{
Claudia María Cárdenas Romero ${ }^{1}$ \\ Dr. Jesús Pedro Miranda Torres ${ }^{2}$
}

\section{RESUMEN}

Se analizan prácticas y significados que los clientes de salones de belleza y peluquerías en Navojoa Sonora otorgan a estos servicios y al cuidado de la apariencia, con base a entrevistas estructuradas. El propósito de esta investigación es descubrir cuáles son los factores que influyen en los clientes al momento de elegir un producto o servicio en las estéticas. Se consideró a la población de Navojoa que reúne ciertas características como la asistencia a salones de belleza y que conozcan sus servicios y productos. De esa población se tomó una muestra de 100 personas tanto hombres como mujeres. Se estructuro un instrumento para encuestar a las personas con los temas relacionados a esta investigación. Todo con el fin de encontrar cuales son los factores más importantes en la elección de los servicios de salones de belleza. Se examina cómo estas elecciones están condicionadas por el posicionamiento social, y cómo contribuyen a producir, reproducir o desestabilizar el género, moduladas por las

\footnotetext{
${ }^{1}$ Instituto Tecnológico de Sonora, klaudia-gasol@ hotmail.com

${ }^{2}$ Instituto Tecnológico de Sonora, jesuspedrot@hotmail.com
} 


\section{http://revistainvestigacionacademicasinfrontera.com}

intersecciones de clase, raza y sexualidad en el contexto de expansión y escasa profesionalización de dichos servicios. Se concluye con el vínculo de estas categorías con la búsqueda de honor y prestigio o la estigmatización. Lo que caracteriza la elección de productos o servicios por sus clientes, es el valor que estos les dan. Lo cual esto lleva a generar alguna consideración o percepción de cómo elegir algún producto o servicio que genere mayor satisfacción en el uso, consumo y goce de los mismos. Los servicios constituyen al mayor sector de crecimiento en el mundo; éste se convirtió en un factor importante y fundamental del crecimiento y una variable clave para la competitividad, de igual manera el fácil acceso a la información de ciertas características de los servicios o productos han permitido que los clientes de hoy estén bien informados.

Palabras clave: Servicio, calidad, precio.

\section{INTRODUCCIÓN Y DESARROLLO}

La imagen externa que dan las personas es una gran fuente de información sobre la personalidad que tiene cada una de ellas. La forma de andar, de vestirse, de peinarse, de maquillarse, los gestos que tienen, la expresión de su mirada, la limpieza personal y el aspecto global que nos ofrece, revelan rasgos más o menos relevantes de su forma de ser. Esto hace que a veces nos hagamos inconscientemente una impresión de una persona nada más con observarla, impresión que, en muchos casos, se corresponde a la realidad, como comprobaremos con el paso del tiempo, cuando ya conocemos a esa persona más profundamente. Para Grönroos (2007) señala que el concepto de servicio es una forma de expresar la idea que la organización tiene al respecto a la manera como se propone resolver ciertos tipos de problemas (de sus clientes) de una manera determinada. Esto significa que el 
Año 10.

Núm. 26
Revista de Investigación Académica sin Frontera

ISSN: 2007-8870

\section{http://revistainvestigacionacademicasinfrontera.com}

concepto de servicio tiene que incluir información sobre: Qué propone hacer a la empresa, cómo debería lograrse y con qué tipos de recursos.

También otro concepto de servicio según Heskett, Sasser, \& Hart (1990), ellos son más concretos y afirman que el concepto de servicio debe consustir en una descripción lo más precisa posible que responda a las siguientes preguntas: ¿Cuáles son los elementos importantes del servicio que vamos a prestar?, ¿Cómo esperamos que esos elementos sean percibidos por el segmento del mercado objetivo? y ¿Qué esfuerzos implica lo anterior en términos del modo como el servicio será diseñado, presentado y comercializado?

La importancia del servicio para Rojas (2002), desempeña un papel vital en el proceso de mantener a los clientes contentos. En bienes de consumo duradero. La importancia de los servicios es que cubren todas las actividades que se pueden ayudar a maximizar la satisfacción del consumidor después que éste ha comprado el producto o el servicio y ha iniciado su uso.

Pocas veces nos damos cuenta de la velocidad con que otros nos etiquetan y clasifican; basta una mirada o el cruce de algunas palabras, para determinar si alguien a quien acabamos de conocer podría ser el ejecutivo que buscamos, si estamos frente a una persona incorrecta o a una persona inteligente, si se trata de un individuo con personalidad complicada o alguien con dificultades para relacionarse.

Entendiendo como servicio el conjunto de prestaciones que el cliente pretende recibir además del producto o servicio básico como consecuencia del precio, imagen y reputación del mismo, es un factor que agrega valor al cliente, se puede decir que el servicio determinar la fidelización del cliente dependiendo de su satisfacción, siendo de gran importancia su análisis, medición, evaluación y mejoramiento. Denton (1991) 


\section{http://revistainvestigacionacademicasinfrontera.com}

Se conoce que existen características, percepciones y consideraciones al elegir un servicio y una de ellas es la calidad que tiene cada uno de estos; ante esto, los clientes son quienes pueden y deben evaluar la calidad del servicio o de algún producto a través de sus experiencias o de compras anteriores que han hecho, por lo ello se define la experiencia como la percepción más importante que cada cliente se va forma sobre el servicio que va recibiendo con forme a los servicios y productos que va adquiriendo, pues dependiendo de esto, el cliente puede cerciorarse si recibió lo que él esperaba y detectar si cubrió sus necesidades y está satisfecho. Horovitz, (1997)

La calidad es la satisfacción de las necesidades y deseos de los clientes cumpliendo con las características de los productos o servicios, esto superando las todas expectativas que tiene el consumidor. Según Palacio \& Ribeiro, (1999) definen la calidad como el conjunto de prioridades y características que definen su aptitud para satisfacer unas necesidades expresadas o implícitas.

Para la Norma ISO 8402 define la calidad como el conjunto de características de una entidad que le confieren la aptitud para satisfacer las necesidades establecidas e implícitas.

Según Garrido (2010), describe que las imagen es la apariencia externa de las personas o las cosas, pero cuando se refiere a imagen personal, el término es mucho más amplio y va más allá de la relación del concepto como la indumentaria, el maquillaje y el cabello.

El producto es mucho más que un objeto. Los clientes compran satisfacciones, no productos. Siguiendo esta idea, podemos definir que el producto como un "conjunto de atributos físicos, de servicio y simbólicos que producen satisfacciones o beneficios al usuario o comprador" Keegan, (1997) 


\section{$\underline{\text { http://revistainvestigacionacademicasinfrontera.com }}$}

Según Soriano (1990), la importancia del producto está en las ventas que tiene éste, el aporte y la participación del mercado. Tambien otro punto es en las implicaciones estratégicas, quwe esto permite mostrar la importancia que cada producto tanto por aportre, el volumen de ventas de la empresa, de la marca, de la decisión, etcétera, como su importancia respecto al mercado, la cual se mide en función de la participacion del mercado de cada producto

La imagen que los demás se formen de nosotros, a partir de una primera impresión, es la que generalmente perdura y la que nos abre o nos cierra las puertas hacia nuevas oportunidades.

Todos los seres humanos proyectamos una imagen frente a los demás, que comienza a formarse a partir de nuestros aspectos físicos, se fortalece con todo aquello que dejamos ver de nosotros y se consolida con lo que los demás perciben e interpretan de cada una de nuestras actuaciones.

El término estética tiene distintas acepciones. En el lenguaje actual se denota en general lo bello, tiene por objeto la esencia y la percepción de la belleza. También se puede referir al campo del arte, y finalmente puede significar la percepción en general. Según Danto (2003) la belleza era considerada de forma unánime como meta suprema del arte y hasta como sinónimo de excelencia artística. Hoy, en cambio, algunos la complementan como algo para recido a un delito estético. En nuestros días, determinada crítica increpa a los artistas cuando sus obras parecen aspirar a la belleza.

Para del Hoyo (2007), las habilidades que conforman el área de cuidado personal incluyen una serie de competencias que hacen referencia al conocimiento y control que los/as niños/as van alcanzando de sí mismos y a la capacidad de utilizar dichos conocimientos en las demandas que la vida diaria nos impone. Las habilidades adaptativas que dan forma al ámbito de aprendizaje que conduce a la autonomía e independencia de cualquier persona, engloba un amplio abanico de destrezas que tienen su origen en el desarrollo senso-perceptivo y motor del 


\section{http://revistainvestigacionacademicasinfrontera.com}

individuo. Algunas personas con graves alteraciones del desarrollo, pueden tener especialmente afectada la capacidad para extraer información del entorno a través del desarrollo sensorial, perceptivo y motriz, dependiendo casi totalmente de la supervisión y guía de otro para empezar a interactuar con el medio, tomar conciencia del mismo y aprender a controlarlo.

Los servicios pueden clasificarse conforme a diversos criterios. Por la índole del servicio, pueden ser servicios personales y productos intangibles. Por el objeto sobre el que se realiza el servicio pueden ser referidos a bienes o referidos a personas. Por el sitio donde se presenta el servicio o forma de distribución pueden ser en la sede del prestador y distribución masiva y por redes electrónicas. Espinosa \& Cervantes Aldana, (2000)

Los salones de belleza tienen diferentes líneas y mezclas de productos según Kotler \& Armstrong (2012), una organización que tiene lineas de producto tiene una mezcla de productos. Una mezcla de productos consiste en todas las lneas de productos y artículos que una empresa determianda ofrece a la venta. Actualmente las empresas dedicadas a la belleza manejan diferentes tipos de productos para las necesidades de la piel, el cabello, faciales, para los pies, etcétera.

Los factores que influyen en la elección de un producto o servicio resulta ser uno de los eventos más importantes en la satisfacción del cliente ya que ha de elegir los servicios y productos a la cual va a tener o conservar por algún tiempo. En relación a esta elección, se han emitido varios conceptos. Entre ellos encontramos uno que consideramos se adecúa más a los aspectos que se abordan en este trabajo de investigación, y consiste en el aporte que a este respecto hace Galindo (2007) que una afirmacion clásica en el mundo comercial es que el producto es el corazón de la mercadotecnia. En verdad, al margen de los mercados en los que 


\section{$\underline{\text { http://revistainvestigacionacademicasinfrontera.com }}$}

por una razon u otra la competencia está restringida, si se analiza desde la perspectiva histórica la trayectoria de las formas próspera y sólidamente implantadas en el mercado hay que concluir que en la inmensa mayoría de los casos su éxito está ligado a la oferta de buenos productos. Ninguna empresa ha mantenido de forma duradera un mal producto en el mercado. No quiere esto decir que un buen producto sea la garantía del éxito, pero sí que un mal producto es el peor cimiento para la construcción de la actividad comercial.

Otros de los conceptos más importantes es el de servicio que para Grönroos en su libro Marketing y gestión de servicios (1990), es una forma de expresar la idea de que la organización se propone resolver ciertos tipos de problemas de una manera determinada. Esto significa que el concepto de servicio tiene que infomracion sobre que se propone hacer en la empresa de un segmento concreto de clientes. El concepto de servicio debe ser lo más concreto posible para que todos lo puedan comprender.

Los servicios pueden clasificarse conforme a diversos criterios. Por la índole del servicio, pueden ser servicios personales y productos intangibles. Por el objeto sobre el que se realiza el servicio pueden ser referidos a bienes o referidos a personas. Por el sitio donde se presenta el servicio o forma de distribución pueden ser en la sede del prestador y distribución masiva y por redes electrónicas. Espinosa \& Cervantes Aldana (2000)

Como antecedentes de los salones de belleza, según Martha Matilde Harper una de las mujeres más reconocidas sobre belleza según el libro Enterprising Women: 250 Years of American Business del autor Drachman (2004) ; en el antiguo Egipto se cuidaba mucho la apariencia física pero no se tenía un lugar específico para practicar sus actividades tales como: los baños de leche, exfoliaciones de la piel, maquillaje en el rostro, manicura, los cortes de cabello, entre otras cosas más simbólicas de la época. Fue hasta en la Antigua Grecia cuando los salones de 


\section{http://revistainvestigacionacademicasinfrontera.com}

belleza comenzaron a tener un lugar en la sociedad, donde solo se peinaban las cabezas más selectas. Esto comenzó a partir del nacimiento de las expresiones artísticas y la vanidad adquirida gracias a las que se empezó a utilizar grasa animal y mezclas de hierbas que recolectaban para crear cremas y maquillajes que eran exclusivos de la clase alta para embellecerse un poco más, dando pauta a todo tipo de soluciones, peinados, joyas y vestimenta.

En la ciudad Navojoa Sonora existen 247 establecimientos de estéticas o bien, salones de belleza según INEGI (2015), que cada uno de ellos ofrecen distintos productos para el cuidado personal, ya sea: tratamientos para el cabello, champú, jabones para el cuerpo, también se ofrecen productos de maquillajes como son; bases, labiales, tratamientos faciales y sombras para ojos, productos que tengan que ver con el aseo personal, etcétera.

También se ofrecen lo que son los servicios, la mayoría de los salones de belleza tiene por igual su menú de servicios que son: cortes de cabello, colorimetría, maquillajes, peinados, soluciones de permanentes, manicure y pedicura, planchados, rizos, ondas y tratamientos aplicados para el cabello.

El objetivo general que se busca de esta investigación es definir que factores son los más importantes para los clientes de Navojoa al momento de elegir los products o servicios de una estética, mediante una aplicación de encuestas esto para saber cuales son las variables más importantes en la elección de ellos y así llegar a su satisfacción.

Como hipótesis de esta investigación:

H1: Los factores que intervienen en las personal al elegir un servicio en salones de belleza son opiniones de las personas, la calidad, el precio y la atención al cliente. 


\section{$\underline{\text { http://revistainvestigacionacademicasinfrontera.com }}$}

H2: Saber si la calidad y el precio son los factores más relevantes en la decisión del cliente.

H3: El trato que proporciona el personal de la estética al momento de estar adquiriendo un servicio o producto se asocian con la satisfacción del cliente.

Todo esto nos lleva al planteamiento del problema de esta investigación, en base a todos los temas anteriores y las investigaciones ya realizadas. Considerando que existe un gran número de estéticas en el municipio de Navojoa, ¿Qué factores influyen en la elección del servicio en una estética en los Navojoenses?.

\section{METODOLOGÍA}

La presente investigación es de tipo cuantitativo, de alcance descriptivo, transversal, con diseño no experimental Münch y Ángeles (2007). Esta investigación es de tipo no experimental ya que se realiza sin la manipulación deliberada de variables y en los que sólo se observa los fenómenos en su ambiente natural para después analizarlos. Así mismo, se realiza un estudio transversal, debido a que se estudia los factores que influyen la elección de servicios en un periodo específico del tiempo, no generalizando ni aplicando los datos presentados a situaciones futuras Münch y Ángeles (2007).

Por otra parte, el alcance de la investigación es descriptivo, es decir, busca especificar cuáles son los factores influyentes en la toma de decisiones de los clientes y rasgos importantes de acuerdo al periodo en el que se efectuará la investigación.

El presente estudio se realizó con una muestra de 100 consumidores, de los cuales el $82 \%$ son mujeres y solamente el $18 \%$ son hombres, mismos que se encuentran en distintos rangos de edad los cuales son de 18 a 34 años en donde se presenta un 49\% de los encuestados y de 35 a 


\section{http://revistainvestigacionacademicasinfrontera.com}

49 años tenemos alrededor del 51\%. De igual manera se encuentran en 2 estados civiles el $65 \%$ es casado y el $35 \%$ soltero.

El instrumento utilizado para la presente investigación, fue diseñado y validado por un maestro investigador y asesor de este proyecto, basándose en temas relacionados a la imagen, a los productos, servicios, la calidad, etcétera. El instrumento de factores que influyen en la elección de servicios está compuesto de 15 preguntas, en donde se hace referencia a la percepción que tiene el consumidor de los factores que se consideran importantes para la elección de algún producto o servicio.

Para las respuestas, se manejó una escala de Likert, con cuatro opciones: 1. Totalmente de acuerdo, 2. De acuerdo, 3. En desacuerdo, 4. Totalmente en desacuerdo. También se manejó escalas, dentro de una escala del 0 a 8 , en donde el 0 era la respuesta con menor resultado y el 8 con el mayor resultado. En cada una de las encuestas se incluye edad, sexo, rangos de edades y situación civil. Así como el objetivo de la encuesta y las instrucciones de la misma.

Una vez seleccionado el instrumento y adecuado al contexto de la investigación, se procedió a la aplicación y tabulación del mismo mediante un paquete estadístico. Finalmente se analizaron cada una de las dimensiones que integran el cuestionario, de tal forma que se asegura la correcta medición de cada variable y permitan su veracidad en la información adquirida para la presentación e interpretación de los resultados.

\section{RESULTADOS}


Núm. 26

ISSN: 2007-8870

\section{$\underline{\text { http://revistainvestigacionacademicasinfrontera.com }}$}

Una vez desarrollada la metodología se continuó con la interpretación de los resultados arrojados de las encuestas, mismos que se obtuvieron en consideración a las percepciones de los clientes que dieron respuesta al cuestionario.

En la cuestión de cuantas veces se adquiere un servicio de salones de belleza se encontró que el $66 \%$ de las personas encuestadas adquieren servicios de una estética cada mes, debido a que existen necesidades por mejorar su apariencia y su estado de ánimo por otra parte; el otro 11\% dice adquirir servicios cada 4 meses o más (Gráfica 1)

Gráfica 1. Cada que tiempo adquieren servicio de estética.

\section{¿Cuántas veces adquiere el servicio de una estética?}
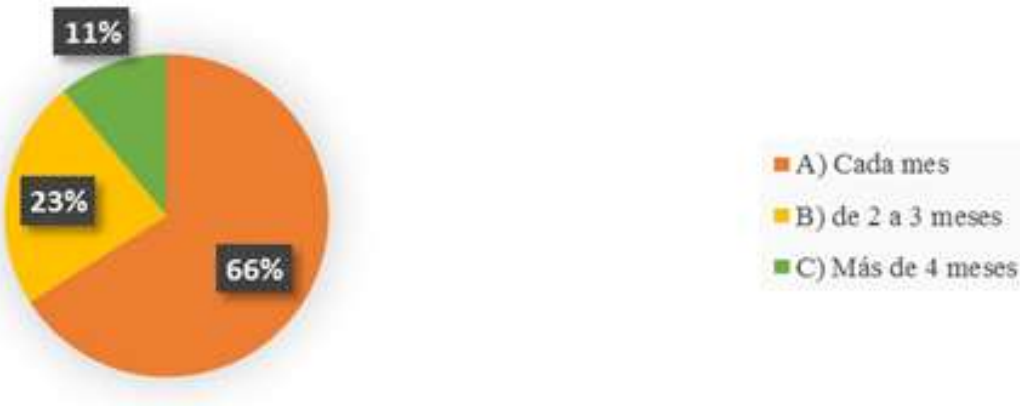

Fuente: Elaborado por autores.

Por otra parte, el 90\% de los encuestados adquiere más el servicio de corte de cabello, un $63 \%$ dice adquirir por servicio de colorimetría y un $43 \%$ utiliza el servicio de maquillaje (Tabla 1).

Tabla 1. Servicios que más se adquieren en una estética.

\begin{tabular}{|l|c|}
\hline $\begin{array}{c}\text { SERVICIOS } \\
\text { DISPONIBLES EN LAS } \\
\text { ESTÉTICAS }\end{array}$ & PORCENTAJES \\
\hline Corte de cabello & $90 \%$ \\
\hline Colorimetría & $63 \%$ \\
\hline Maquillaje & $43 \%$ \\
\hline
\end{tabular}




\section{http://revistainvestigacionacademicasinfrontera.com}

\begin{tabular}{|l|l|}
\hline Peinado & $33 \%$ \\
\hline Manicure y pedicura & $26 \%$ \\
\hline Ceja & $22 \%$ \\
\hline Barba & $14 \%$ \\
\hline Gelish & $30 \%$ \\
\hline Otros & $16 \%$ \\
\hline
\end{tabular}

Fuente: Elaborada por los autores.

De las personas encuestadas, consideraron varios factores en la toma de decisión del servicio de una estética. El 84\% contestaron que uno de los factores más importantes para la elección de un servicio o producto de un salón de belleza es la calidad de los productos y servicios que tiene cada establecimiento, mismos con un $80 \%$ consideran que el precio es factor importante en la toma de decisión y por último un 50\% perciben que la opinión de las personas se considera mucho al momento de elegir en que salón de belleza poner sus manos (Tabla 2).

Tabla 2. Factores que influyen en la elección de un servicio o producto en una estética

\begin{tabular}{|l|c|}
\hline $\begin{array}{c}\text { FACTORES QUE INFLUYEN EN } \\
\text { LA ELECIÓN DE UN SRVICIO O } \\
\text { PRODUCTO }\end{array}$ & PORCENTAJES \\
\hline Precio & $80 \%$ \\
\hline Calidad & $84 \%$ \\
\hline Atención al cliente & $23 \%$ \\
\hline Exclusividad en productos y servicios & $22 \%$ \\
\hline Opiniones de las personas & $50 \%$ \\
\hline Comodidad y facilidad & $31 \%$ \\
\hline
\end{tabular}

Fuente: Elaborada por los autores.

En cuanto a la comparación de precios de los productos y servicios de los distintos establecimientos. Se puede definir que el $41 \%$ de las personas consideran estar de acuerdo en comparar precios con distintas empresas para su mayor satisfacción y un $22 \%$ menciona que no compara precios (Gráfica 2).

Gráfica 2. Comparar precios de productos. 
Año 10.

Núm. 26
Académica sin Frontera

ISSN: 2007-8870

\section{http://revistainvestigacionacademicasinfrontera.com}

\section{Siempre comparo los precios de los productos al momento de comprar}

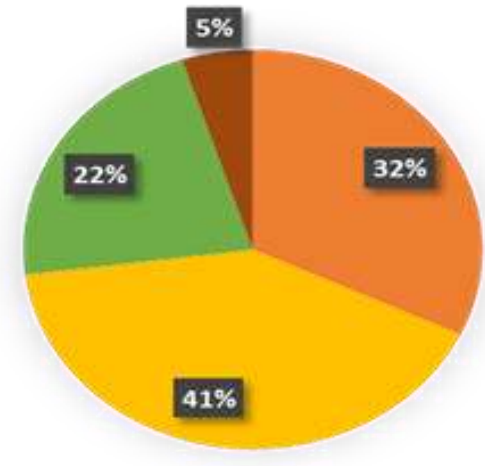

$$
\begin{aligned}
& \text { = A) Totalmente de acuedo } \\
& =\text { B) De acuerdo } \\
& \text { =C)Totalmente en desacuerdo } \\
& \text {-D) En desacuerdo }
\end{aligned}
$$

Fuente: Elaborado por los autores.

El $40 \%$ considera en adquirir un servicio de estética en otro establecimiento si no está disponible el que regularmente se dirige (Gráfica 3).

Gráfica 3. Adquirir servicios de otras estéticas.

\section{Adquiero un servicio en otra estética si no está} disponible en la que regularmente voy?

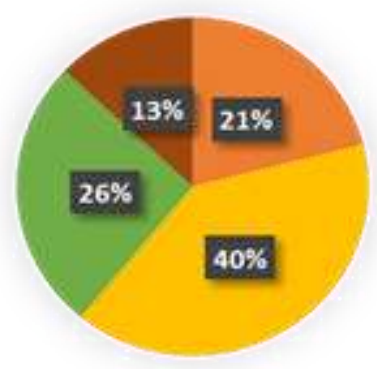

$$
\begin{aligned}
& \text { = A) Totalmente de acuedo } \\
& \text { =B) De acuerdo } \\
& \text { =C)Totalmente en desacuerdo } \\
& \text {-D) En desacuerdo }
\end{aligned}
$$

Fuente: Elaborado por los autores. 


\section{http://revistainvestigacionacademicasinfrontera.com}

Por otro lado, se consideró preguntarles a las personas si se fijan en los precios más bajos al adquirir algún servicio o producto en los salones de belleza. El 48\% considera que siempre se fija en el precio más bajo al adquirir algún servicio o producto en un salón de belleza. Pero el $27 \%$ no considera que exista importancia a buscar precios más bajos (Gráfica 4).

Gráfica 4. Fijarse en precios más bajos.

\section{Siempre me fijo en el precio más bajo cuando elijo a que estética ir?}
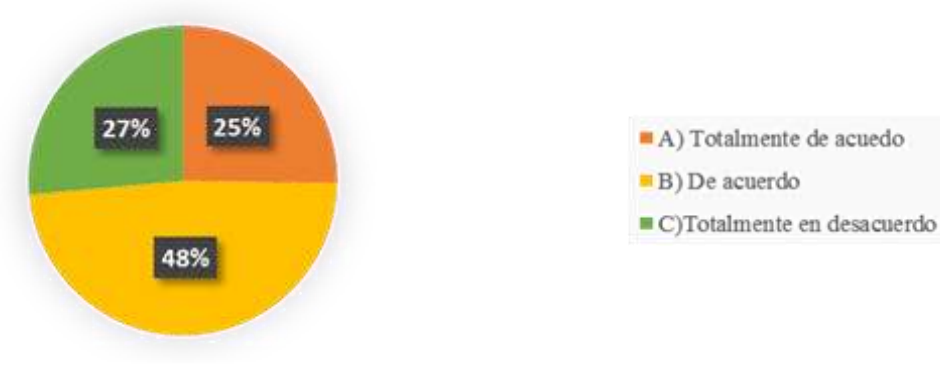

Fuente: Elaborado por los autores.

Las mujeres encuestadas calificaron marcas de maquillajes y de tintes que conocen y que utilizan más considerando 8 siendo el más utilizado y el 0 menos utilizado. Teniendo así un resultado que la marca de producto de belleza "Maybeline" es la que se consume más considerándole con la calificación más alta, seguida con la marca "Bissú" y por ultimo las marcas "E.L.F” y "Clinique". Estas marcas son las que se consideran más utilizadas por las personas encuestada. (Tabla 3).

Tabla 3. Resultados de marcas de maquillajes más utilizadas.

\begin{tabular}{|l|c|}
\hline \multicolumn{1}{|c|}{ MARCAS DE MAQUILLAJE } & $\begin{array}{c}\text { RESULTADOS ENUMERACIÓN DEL 1 } \\
\text { AL 8, SIENDO 8 LA CALIFICACIÓN } \\
\text { MAYOR }\end{array}$ \\
\hline Mehron & 4 \\
\hline
\end{tabular}


Revista de Investigación

Año 10. Académica sin Frontera

Núm. 26

ISSN: 2007-8870

\section{http://revistainvestigacionacademicasinfrontera.com}

\begin{tabular}{|l|c|}
\hline Bissú & 7 \\
\hline E.L.F & 6 \\
\hline Maybeline & 8 \\
\hline LA Girl & 3 \\
\hline Cyzone & 0 \\
\hline Clinique & 5 \\
\hline Revlon & 2 \\
\hline Otro & 1 \\
\hline
\end{tabular}

Fuente: Elaborado por los autores.

De igual manera, las marcas de tintes "Hidracolor" y "L'Oréal" sobresalieron con las calificaciones más altas de la encuesta. Siendo así las marcas que más se utilizan en la localidad (Tabla 4).

Tabla 4. Resultados de marcas de tintes más utilizados.

\begin{tabular}{|l|c|}
\hline \multicolumn{1}{|c|}{ MARCAS DE TINTES } & $\begin{array}{c}\text { RESULTADOS ENUMERACIÓN } \\
\text { DEL 1 AL 8, SIENDO 8 LA } \\
\text { CALIFICACIÓN MAYOR, }\end{array}$ \\
\hline L'Oréal & 6 \\
\hline Color and art & 5 \\
\hline Kunl & 2 \\
\hline By Fama & 1 \\
\hline Garnier & 3 \\
\hline Koleston & 4 \\
\hline Hidracolor & 7 \\
\hline Otro & 0 \\
\hline
\end{tabular}

Fuente: Elaborado por los autores.

El $49 \%$ de las personas encuestadas se dirige a cortarse el cabello a una estética cada mes, un $42 \%$ considera que cada dos meses va y por ultimo un $9 \%$ de los encuestados dice que cada 4 meses (Gráfica 5). 
Núm. 26

ISSN: 2007-8870

http://revistainvestigacionacademicasinfrontera.com

Gráfica 5. Cada que tiempo se dirigen a cortase el cabello.

\section{Con que frecuencia va a cortarse el pelo?}
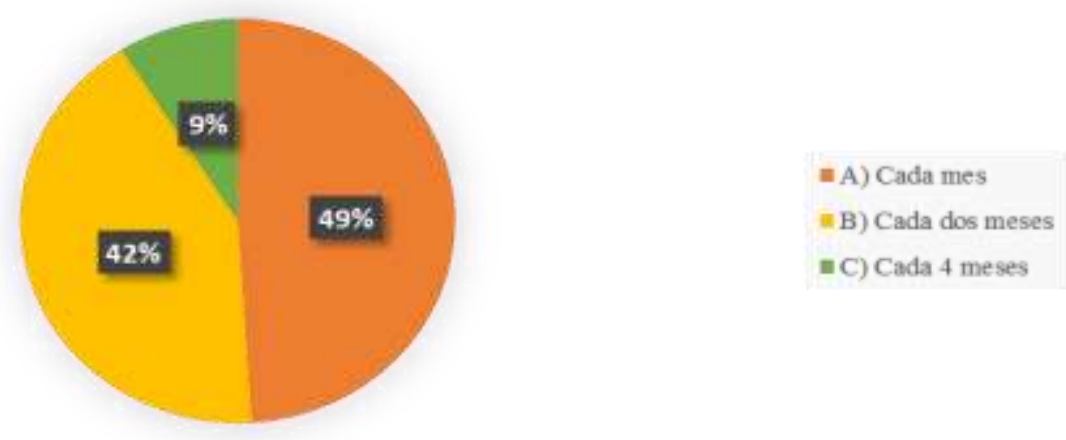

Fuente: Elaborado por los autores.

Las personas que se tomaron para encuestarlas van con frecuencia a un salón de belleza para teñirse el cabello. Un $53 \%$ adquiere el servicio de colorimetría cada dos meses, el $32 \%$ cada mes y el otro $15 \%$ se dice que lo adquiere cada 4 meses (Gráfica 6).

Gráfica 6. Con qué frecuencia se dirigen a teñirse el cabello.

\section{Con qué frecuencia se dirige a pintarse el cabello?}
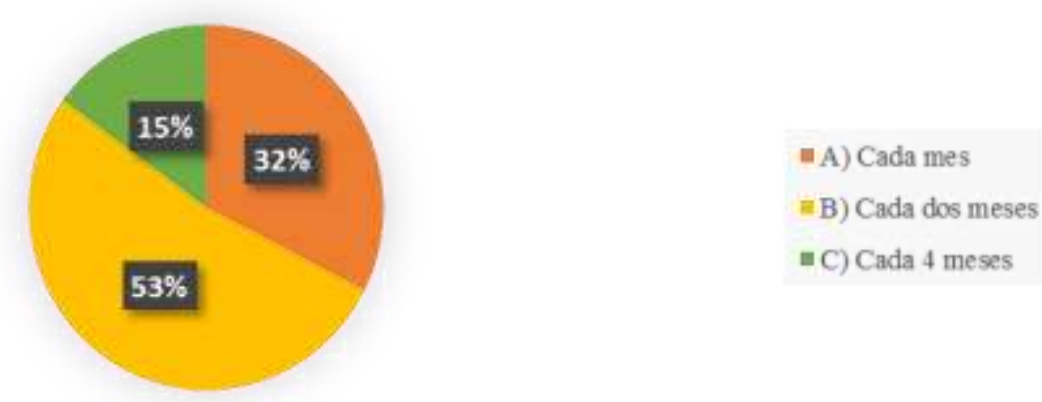

Fuente: Elaborado por los autores. 
Núm. 26

ISSN: 2007-8870

\section{http://revistainvestigacionacademicasinfrontera.com}

Finalmente, a las personas encuestadas se les preguntó su edad, sexo y estado civil como anteriormente se dijo. Un $82 \%$ de las personas encuestadas son mujeres, ya que son las personas que más utilizan estos servicios y productos y solamente el $18 \%$ son hombres (Gráfica 7).

Gráfica 7.-Género de las personas encuestadas.

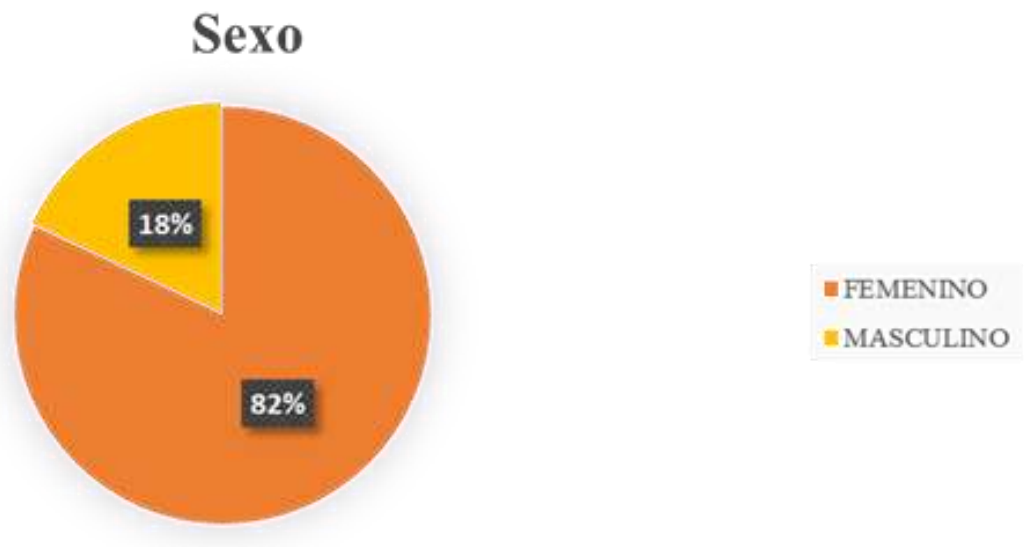

Fuente: Elaborado por los autores.

De esos encuestados, tenían un rango de edades entre los 18 a 34 años y los 35 a 49 años. Los resultados obtenidos de edad, fueron que el $51 \%$ de las personas encuestadas son del rango de edades de los 35 a los 49 años, y el resto que es un 49\% son del rango de 18 a 34 años de edad. (Gráfica 8).

Gráfica 8. Rango de edades de los encuestados 
Año 10. Académica sin Frontera

Núm. 26

ISSN: 2007-8870

http://revistainvestigacionacademicasinfrontera.com

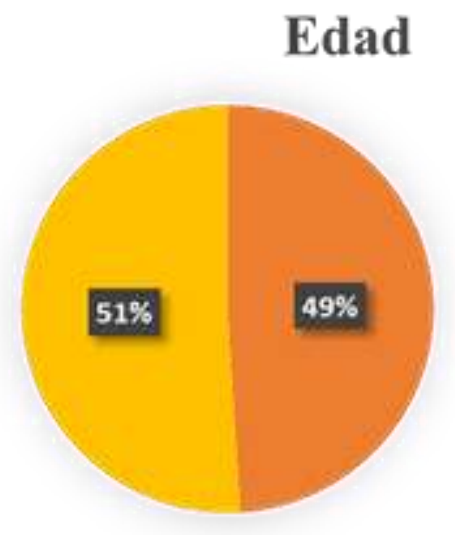

a) 18-34 años

- $\mathrm{B}) 35-49$ aกीos

Fuente: Elaborado por los autores.

Y por último, el estado civil de cada persona encuestada. El 65\% de las personas encuestadas son casadas y el $35 \%$ están en estado civil soltero (Gráfica 9).

Gráfica 9. Estado civil de los encuestados.

\section{Estado civil}

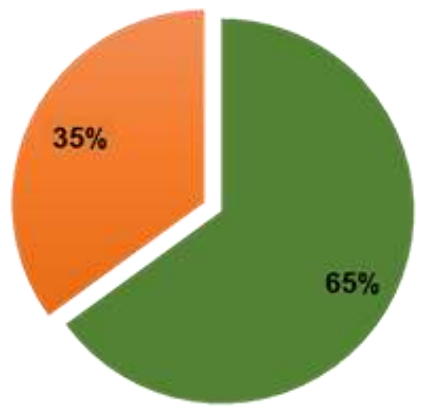

Fuente: Elaborado por los autores. 


\section{$\underline{\text { http://revistainvestigacionacademicasinfrontera.com }}$}

Actualmente en el mundo las personas se preocupan más por su aspecto físico y la apariencia, las organizaciones están obligadas a mostrar un mayor interés en los clientes y atraerlos, ya que por ellos son los que siguen en pie las empresas.

Los resultados permiten plantear cuales son los elementos evaluados con los que las personas consideran elegir un producto o servicio en las estéticas o salones de belleza.

La investigación realizada en los clientes de las distintas estéticas y salones de belleza en Navojoa Sonora para la saber cuáles son los factores que más influyen en la elección de un servicio o producto, se encontró que el factor con más valor que se considera importante es la calidad, ya que esta es una variable importante en el mercado, es un valor con más peso al momento de seleccionar algún producto y para la satisfacción del cliente.

La calidad en las empresas es muy importante ya que es una de las propiedades inherentes a algún producto o servicio que esta permite caracterizarla y valorarla respecto a las restantes de su especie; consideran que en algunas empresas dedicadas a la belleza no cuentan con calidad suficiente en sus servicios y también en los productos que brindan como venta.

Otro de los factores más sobresalientes en esta investigación es el precio, ya que todos los productos y servicios que se ofrecen en el mercado tienen un costo. Muchos de los consumidores consideran que el precio es algo relevante en lo que se consume.

Se considera comparar precios con la competencia, buscar precios más bajos o accesibles al bolsillo.

Y por último, en el caso de salones de belleza, otro factor importante es la opinión de las personas. Como son establecimientos dedicados a la belleza, la opinión de las personas en 


\section{http://revistainvestigacionacademicasinfrontera.com}

cuanto al servicio es de mayor importancia, ya que es algo donde ellos confiarán su imagen y la percepción que los demás tengan de su apariencia.

\section{CONCLUSIÓN Y RECOMENDACIÓN}

Estamos viviendo en tiempos no sofisticados en los que cada quien establece su propio estilo de vida, lo que embarca la vestimenta, la forma de arreglarse, etcétera, muchas veces alejado de lo que manda la etiqueta; personajes públicos, del mundo empresarial y especialmente del artístico, asisten a toda clase de actos y eventos, ataviados de las más variadas maneras, reforzando o deteriorando la imagen personal que cada cual ha querido mostrar.

De acuerdo con la siguiente investigación se puede evidenciar que se encontró el resultado de la hipótesis 1, que los factores más influyentes en la elección de servicios en las estéticas, siendo las de mayor incidencia en los resultados, se encontró; La calidad, ya que los consumidores toman importancia en el aspecto que tenga el producto o servicio, utiliza sus sentidos y su propia experiencia para evaluar sí un producto o servicio es de buena o mala calidad en función de sus expectativas. Claro, también toma prestada la experiencia ajena que muchas veces asimila como propia. Esto nos lleva a otro factor que es la opinión de las demás personas. De igual manera, otro factor importante fue el precio, con relación a este factor queda marcado el valor del producto o servicio, por lo tanto un producto de calidad final inferior pero con un precio mucho menor que el de los competidores termina generando un mayor valor agregado para el cliente, que es lo que finalmente cuenta. Con esto se concluye que las hipótesis 2 y 3 también tienen un resultado, siendo la H2 que la calidad y el precio si son factores relevantes al momento de elegir algún servicio o producto en un salón de belleza y la H3 que el buen trato del personal de las estéticas al estar adquiriendo un servicio se asocia con la satisfacción que tiene el cliente. 


\section{http://revistainvestigacionacademicasinfrontera.com}

Conocer el los factores que influyen en nuestra decisión de adquirir algo es relevante para cualquier empresa conocer el cual es lo más importante de su producto, considerando que si existe factores favorable va a condicionar una mayor calidad en las empresas, cabe descartar que en México los factores influyentes son importantes por su contribución al crecimiento de las organizaciones.

Bibliografía

Danto, A. C. (2003). El Abuso de la Belleza. La estética y el concepto del arte.. Barcelona: Paidós. p. 240

Denton, D. K. (1991). Calidad en el Servicio a los Clientes. Madrid España: Ediciones Díaz de Santos. p. 1

Drachman, V. G. (2004). Enterprising Women: 250 Years of American Business. Los Ángeles. p.93

Espinosa, J. S., \& Cervantes Aldana, J. (2000). Mercadotecnia de servicios. Contaduría y Administración, p. 199. Obtenido de Mercadotencia de servicios.

Galindo, J. L. (2007). Claves para gestionar precio, producto y marca. España: Wolters Kluwer. p. 15

Garrido, R. A. (2010). Imagen Personal. Málaga: Editorial Vértice. p.18

Geografía, I. N. (25 de Enero de 1983). INEGI. Obtenido de http://www.inegi.org.mx/

Grönroos, C. (2007). Service Management and Marketing. Illustrated. p. 112

Heskett, J. L., Sasser, W. E., \& Hart, C. (1990). Service Breakthroughs. New York. p. 24

Grönroos, C. (1990). Marketing y gestión de servicios. España: Díaz de Santos. p.112

Horovitz, J. (1997). La Caidad del Servicio. A la Conquista del Cliente. Madrid: Editorial Mc Graw Hill. p.2 


\section{http://revistainvestigacionacademicasinfrontera.com}

Hoyo, I. d. (2007). Cuidado Personal Universidad Autónoma de Madrid. Madrid.

Keegan, W. (1997). Marketing Global: Quinta edición. Prentice Hall. p.15

Kotler, P., \& Armstrong, G. (2012). Fundamentos de Marketing. México: Pearson. p.305

Münch, L., \& Ángeles, E. (2007). Métodos y técnicas de investigación. México: Editorial Trillas S.A. de C.V. p.23

Normalización, O. I. (1947). Norma ISO 8402. Ginebra.

Palacio, J. R., \& Ribeiro, S. D. (1999). Creación y Dirección de PYMES. Madrid: Ediciones Díaz de Santos. p. 247

Rojas, F. A. (2002). Consumidor Clientela y Distribución para la Economía del Futuro. Madrid: ESIC.p. 75

Soriano, C. N. (1990). Instrumentos de Análisis del Marketing Estratégico. Madrid España: Díaz de Santos. p.51 